\title{
Malaria and gravidity interact to modify maternal haemoglobin concentrations during pregnancy
}

Smailla Ouédraogo ${ }^{1,3,4^{*}}$, Florence Bodeau-Livinec ${ }^{1,2,4}$, Valérie Briand ${ }^{1,4}$, Bich-Tram Huynh ${ }^{1,4}$, Ghislain K Koura ${ }^{1,4}$, Manfred MK Accrombessi ${ }^{3}$, Nadine Fievet ${ }^{1,4}$, Achille Massougbodji ${ }^{3}$, Philippe Deloron ${ }^{1,4}$ and Michel Cot $^{1,4}$

\begin{abstract}
Background: Primigravidity is one of the main risk factors for both malaria and anaemia. Since the implementation of intermittent preventive treatment (IPTp) in sub-Saharan Africa, the relationship between anaemia and gravidity and its evolution during pregnancy has been little explored. This study aimed to evaluate the impact of gravidity on the variation of haemoglobin during pregnancy according to the timing of gestation.

Methods: Data from three studies carried out in nearby areas in south Benin (Ouidah, Comé, Allada) between 2005 and 2012 were analysed. At inclusion (first antenatal visit, ANV1) women's age, area of residence, schooling, gravidity, gestational age, weight and height were recorded. Thick blood smears were performed on ANV1, second visit (ANV2) and at delivery. In Allada, women's serum ferritin and CRP concentrations were also assessed. The impact of gravidity on maternal haemoglobin $(\mathrm{Hb})$ was analysed using a logistic or linear regression depending on the outcome. The statistical significance was set to $P<0.05$.

Results: In total, data from 3,591 pregnant women were analysed. Both univariate and multivariate analyses showed a constant association between $\mathrm{Hb}$ concentrations and gravidity in the three periods of $\mathrm{Hb}$ assessment (ANV1, ANV2 and delivery). Mean $\mathrm{Hb}$ concentration was significantly lower in primigravidae than in multigravidae at ANV1 (mean difference $=-2.4 \mathrm{~g} / \mathrm{L}, \mathrm{Cl} 95 \%$ : $[-3.4,-1.4], \mathrm{P}<0.001$ ). Afterwards, there was a significant increase in primigravidae only, with a tendency to reversal between primigravidae and multigravidae, which was confirmed at delivery (mean difference $=2.8 \mathrm{~g} / \mathrm{L}, \mathrm{Cl} 95 \%$ : $[1.3,4.2], \mathrm{P}<0.001$ ). The prevalence of malaria infection was halved between ANV1 and delivery in primigravidae while it decreased by only $38 \%$ among multigravidae, who were less prone to malaria infection (prevalence at ANV1, 20\% and 10\% respectively). Iron deficiency was more common in multigravidae, and it decreased slightly in this group between ANV1 and delivery.
\end{abstract}

Conclusion: In a context of IPTp, Hb levels improved progressively throughout pregnancy in primigravidae, likely as a result of reduction in malaria infection. In multigravidae, the improvement was less perceptible and anaemia was mainly due to iron deficiency.

Keywords: Anaemia, Gravidity, Malaria, Iron deficiency, Prevention

\section{Background}

As part of the prospective cohort study (Anaemia in Pregnancy: Etiologies and Consequences "APEC") carried out in Benin between 2010 and 2012, primigravidae and multigravidae were followed from the first antenatal visit (ANV1) until delivery. In a preceding paper [1],

\footnotetext{
* Correspondence: smaila11@yahoo.fr

${ }^{1}$ Mère et enfant face aux infections tropicales, IRD Unité mixte de recherche 216, Paris, France

${ }^{3}$ Laboratoire de Parasitologie, Faculté des Sciences de la Santé, Cotonou, Benin

Full list of author information is available at the end of the article
}

analyses showed that the administration of anti-parasitic treatments (IPTp with SP or mefloquine and albendazole) at ANV1 significantly decreased the prevalence of malaria infection and helminths. Furthermore, these analyses showed that malaria and helminth infestations were no longer associated with anaemia at the second antenatal visit (ANV2). At ANV2, the effect of primigravidity on anaemia, particularly marked at enrolment, also disappeared, suggesting this group could be particularly sensitive to preventive measures.

First pregnancy is recognized as one of the main risk factors for both malaria and anaemia [2-4]. The

\section{Ciomed Central}


variations of haemoglobin $(\mathrm{Hb})$ levels throughout pregnancy have seldom been investigated, although an early study in Papua New Guinea, where pregnant women received neither malaria prophylaxis nor haematinics, reported that the differences in $\mathrm{Hb}$ levels between malaria-positive at booking and malaria-negative at delivery were higher in primigravidae than in multigravidae [5].

However, since the implementation of IPTp in subSaharan Africa, no clinical trial has studied the effect of malaria-focused preventive measures on anaemia in relation to gravidity. To evaluate the impact of gravidity on the variation of $\mathrm{Hb}$ during pregnancy according to the timing of gestation, data from APEC were analysed and further pooled with two earlier studies, which had followed pregnant women in nearby areas in south Benin (Ouidah and Comé).

\section{Methods}

\section{Ethical considerations}

The three studies were approved by the Ethics Committees of the Research Institute for Development in France, and of the Faculty of Medicine of Cotonou in Benin. Before each inclusion, written informed consent was obtained from all participants.

\section{Study sites and population}

The study sites and populations have been described elsewhere [6-8]. Briefly, the three studies were conducted in three rural or semi-rural areas located within a $50 \mathrm{~km}$ radius in south Benin (Ouidah, Come, Allada). In the whole area, malaria is perennial and Plasmodium falciparum is the commonest species. There are two high transmission peaks from April to July and October to November. The study population was composed of HIV-negative pregnant women who attended one of the nine study maternity clinics (three in each site) during the period of the studies.

\section{Ouidah study}

This study is a randomized controlled clinical trial, which took place from 2005 to 2008 in Ouidah, a 35,000inhabitant semi-rural area located $40 \mathrm{~km}$ west of Cotonou, in three maternity clinics (Kindji, Kpassé, Hopital de Zone). It compared the efficacy of sulphadoxinepyrimethamine SP (1,500/75 $\mathrm{mg}$ per dose) vs mefloquine (15 mg/kg per dose) given for IPTp on birth weight. The first IPTp dose was administered between 16 and 28 weeks, and the second dose after 30 weeks of gestation, with at least one month from the first dose intake [6].

\section{STOPPAM study (strategy to prevent pregnancy- associated malaria)}

STOPPAM is a cohort study of pregnant women, set up in three maternity clinics (Comé central, Ouedeme-
Pedah, Akodeha) in the district of Comé, with a population of 58,396 inhabitants. The district of Comé is a rural area located $70 \mathrm{~km}$ west of Cotonou. STOPPAM aimed to investigate the development of immune response to malaria in pregnancy. This study was carried out between 2008 and 2010. Two doses of SP-IPTp were administered according to the national guidelines, the first dose of IPTp being administered between 16 and 24 weeks of gestation. Women were followed up monthly from inclusion to delivery [7].

\section{APEC study (anaemia in pregnancy: etiology and consequences)}

APEC is an observational cohort study nested in MiPPAD (Malaria in Pregnancy Preventive Alternative Drugs), a randomized trial comparing the efficacy of IPTp with SP (1,500/75 mg per dose) and mefloquine (15 mg/kg per dose). APEC took place in three maternity clinics in the district of Allada (Allada, Attogon, Sékou), between 2010 and 2012. The district of Allada is a semi-rural area of 91,778 inhabitants located $50 \mathrm{~km}$ north of Cotonou. As in the Ouidah study, the first dose of IPTp was administered between 16 and 28 weeks of gestation [8].

In the three studies, women were encouraged to consult in case of symptoms any time between scheduled ANVs. Women were systematically given supplements of oral ferrous sulfate (200 mg per day) and folic acid (5 mg per day) for home treatment, according to the guidelines of the Beninese Ministry of Health $(\mathrm{MoH})$ in APEC and STOPPAM studies. In the Ouidah study, the daily dose of ferrous sulphate was $400 \mathrm{mg}$ and folic acid was unchanged ( $5 \mathrm{mg}$ per day). In the three studies, iron and folic acid prescription was renewed if necessary. Theoretically, women were supposed to continue the supplementation until three months after delivery. In cases of $\mathrm{Hb}$ concentration below $110 \mathrm{~g} / \mathrm{L}$, women were treated according to the severity of anaemia, i e, $200 \mathrm{mg}$ of oral ferrous sulphate and $5 \mathrm{mg}$ of oral folic acid twice a day for mild and mode anaemia, and referred to the tertiary hospital of the district in case of severe anaemia $(\mathrm{Hb}<70 \mathrm{~g} / \mathrm{L})$. In the latter case, $500 \mathrm{ml}$ of whole blood were transfused. The total amount of blood transfusion was raised to $1,500 \mathrm{ml}$ if necessary, according to the guidelines of the Beninese $\mathrm{MoH}$.

\section{Socio-demographic and clinical data collection}

At inclusion (ANV1), socio-demographic data such as age, area of residence, level of education were recorded. Women were clinically examined and parity, gestational age, weight and height were recorded. Weights were measured to the nearest $0.1 \mathrm{~kg}$ by using an electronic scale (Seca Corp., Hanover, MD) and heights to the nearest $0.1 \mathrm{~cm}$ by using a bodymeter device (Seca ${ }^{\circledR} 206$ Bodymeter; Seca Corp.). Weights and heights were 
measured by two nurses, and the mean of the two measurements was calculated for all participants. Body mass index (BMI) was defined as the women's weight at enrolment $(\mathrm{kg})$ /height squared $\left(\mathrm{m}^{2}\right)$. Medical history, including the history of previous pregnancies, notation of any chronic disease (as high blood pressure, diabetes, or asthma) was also recorded.

At the time of the second antenatal visit (ANV2), at delivery and during the unscheduled visits, gestational age, weight and height were measured. Gestational age was estimated using the date of the last menstrual period and/or by measuring the fundal height.

\section{Blood sample collection}

At ANV1, ANV2 and at delivery, venous blood was collected from each participant to determine $\mathrm{Hb}$ concentrations and to perform thick blood smears in search of peripheral malaria. At delivery, a placental blood smear was also performed to look for placental malaria. In the APEC study, blood was collected into dry tubes to determine women's serum ferritin and CRP concentrations. Serum ferritin and C-reactive protein (CRP) concentrations were used to evaluate women's iron stores. Iron deficiency was defined as serum ferritin $<12 \mu \mathrm{g} / \mathrm{L}$ or as serum ferritin between 12 and $70 \mu \mathrm{g} / \mathrm{L}$ in the context of inflammation defined as a positive CRP, i.e., CRP concentration $>5 \mathrm{mg} / \mathrm{mL}$.

\section{Laboratory tests}

The laboratory methods have been described elsewhere [6-8]. Haemoglobin concentration was measured with either with a haematology analyser (Erma Laboratory, Japan) or Hemo_Control ${ }^{\circledR}$ (EKF Diagnostics, Germany) device.

In the Ouidah randomized trial and STOPPAM, blood smears were performed using the standard method. Thick blood smears were stained with Giemsa and read at x100 oil immersion. Smears were considered negative if no asexual stage of malaria parasite was detected after counting 500 leucocytes. Malaria parasites were counted against 200 leucocytes [9].

In APEC, the Lambaréné technique was used. It consists of spreading $10 \mu \mathrm{L}$ of blood on a slide's rectangular area of $1.8 \mathrm{~cm}^{2}(1.8 \mathrm{~cm} \mathrm{x} 1 \mathrm{~cm})$. The slide is then stained with Giemsa and read at x100 oil immersion. A multiplication factor is applied to the average parasitaemia/field to get a number of parasites/ $\mu \mathrm{L}$. The Lambaréné method detection threshold has been estimated to five parasites/ $\mu \mathrm{L}$ [10]. Serum ferritin was measured by using an AxSym Immuno-Assay Analyzer (Abbott Laboratories, Abbott Park, IL) with $500 \mathrm{~mL}$ of serum. The concentrations of serum CRP were determined by using a rapid slide test (CRP Latex; Cypress Diagnostics Inc., Campbellville, Ontario, Canada).

\section{Statistical analysis}

Data were analysed with Stata version 11 for Windows (Stata Corp, College Station, TX, USA).

\section{Definition of outcome variables}

Maternal $\mathrm{Hb}$ status was considered first as a continuous variable, and then as a categorical variable. Anaemia was defined as $\mathrm{Hb}$ below $110 \mathrm{~g} / \mathrm{L}$. Severe, mode and mild anaemia were defined as $\mathrm{Hb}$ concentrations less than $70 \mathrm{~g} / \mathrm{L}$, between 70 and $99 \mathrm{~g} / \mathrm{L}$ and between $100 \mathrm{~g} / \mathrm{L}$ and $109 \mathrm{~g} / \mathrm{L}$, respectively [11].

\section{Comparison of the three studies}

Baseline characteristics of the women and the outcomes (maternal $\mathrm{Hb}$ concentrations and maternal anaemia at each blood assessment) were compared between the three studies. Other anaemia risk factors such as malaria, placental malaria and the timing of IPTp intakes were also compared. Proportions and means were compared using Chi2 test and Student's test.

\section{Effects of gravidity on maternal haemoglobin concentrations and maternal anaemia}

To determine the impact of gravidity on maternal $\mathrm{Hb}$ status, analyses were first separately performed on the data from each study. Afterwards, the data from the three studies were pooled to perform a logistic or linear regression depending on the outcome (binary or continuous).

To account for the timing of anti-malarial interventions, two variables measuring the intervals between IPTp1 and IPTp2, and IPTp2 and the end of the pregnancy were created. All variables with a $P$ value less than 0.2 were considered as covariates for the multivariate linear or logistic regressions. The statistical significance of final models was set to $\mathrm{P}<0.05$.

\section{Results}

\section{Comparison of women's general characteristics between} the three studies

Three thousand, five hundred and ninety one $(3,591)$ women were included in this analysis. One thousand, six hundred and one (1601) women were from the Ouidah trial, 985 women from STOPPAM and 1,005 women from APEC. Haemoglobin concentrations were assessed in $98 \%(72 / 3591)$ of the women at ANV1 and $89.4 \%$ $(335 / 3164)$ at delivery.

The general characteristics of women and the outcomes of the three studies are presented in Table 1 . The proportion of women who received education was higher in Ouidah than in STOPPAM and APEC $(\mathrm{P}<0.001)$. The proportions of women less than 21 years old (median age) did not differ between the three studies $(\mathrm{P}=0.22)$, but there were more primigravidae in Ouidah than in STOPPAM and APEC $(\mathrm{P}<0.001)$. The overall 
nutritional status of the mothers, assessed by the body mass index (BMI) on inclusion did not differ significantly between the three studies $(P=0.07)$, but there was a tendency for a lower BMI in Ouidah. Placental malaria was more common in STOPPAM and APEC than in Ouidah (11.2\%, 9.2\% and 3.0\% respectively).

On average, women were given first and second doses of IPTp earlier in STOPPAM and APEC than in Ouidah $(P=0.001)$, but there was no significant difference between gestational ages at delivery $(\mathrm{P}=0.15)$. The time intervals between IPTp intake was highest in Ouidah whereas the interval between IPTp2 and delivery was less in Ouidah than in STOPPAM and APEC. Mean $\mathrm{Hb}$ was higher at each blood assessment in Ouidah than in STOPPAM and APEC, and in the three studies there was a marked tendency to an increase in Hb concentrations from ANV1 to ANV2 and delivery, as described previously for APEC and the Ouidah trial [1,12]. For these reasons, we decided to include the factor "study" as an adjustment covariate in the multivariate analyses.

Twenty point five percent $(20.5 \%)$ of the women had inflammation (CRP > $5 \mathrm{ml} / \mathrm{ml}$ ) at ANV1 and 34.3\% at delivery. The geometric means of malaria parasite density were 601.8 (C195\%: [544.6-735.1]) at ANV1 and 3294.5 (CI95\%: [2208.3-4447.1]) at delivery.

\section{Relationship between maternal haemoglobin status} and gravidity throughout pregnancy

When considering studies separately, there was an overall suppress differences between primigravidae and

Table 1 Comparison of women's general characteristics between the three studies

\begin{tabular}{|c|c|c|c|c|}
\hline Factors & Ouidah study $n=1601$ & STOPPAM $\mathrm{n}=985$ & APEC $n=1005$ & $P$ value \\
\hline Area & Semi-rural & Rural & Semi-rural & \\
\hline \multicolumn{5}{|l|}{ Education of the woman (\%) } \\
\hline None & 44.5 & 56.3 & 66.6 & \multirow[t]{2}{*}{$<0.001$} \\
\hline Some & 55.5 & 43.7 & 33.4 & \\
\hline \multicolumn{5}{|l|}{ Age (\%) } \\
\hline$<21$ & 24.7 & 22.3 & 25.6 & \multirow[t]{2}{*}{0.22} \\
\hline$\geq 21$ & 75.3 & 77.7 & 74.4 & \\
\hline \multicolumn{5}{|l|}{ Gravidity (\%) } \\
\hline Primigravidae & 26.9 & 18.7 & 18.9 & \multirow[t]{2}{*}{$<0.001$} \\
\hline Multigravidae & 73.1 & 81.3 & 81.1 & \\
\hline \multicolumn{5}{|l|}{$\mathrm{BMI}$ at inclusion $\left(\mathrm{kg} / \mathrm{m}^{2}\right)(\%)$} \\
\hline$<20$ & 81.0 & 78.1 & 77.7 & \multirow[t]{2}{*}{0.07} \\
\hline$\geq 20$ & 19.0 & 21.9 & 22.3 & \\
\hline Interval between ANV1 and ANV2 (weeks) mean (SD) & $8.6(3.2)$ & $5.1(1.5)$ & $6.4(1.8)$ & 0.0001 \\
\hline Interval between ANV2 and delivery (weeks) mean (SD) & $8.6(3.3)$ & $13.9(3.9)$ & $12.2(5.4)$ & 0.0001 \\
\hline Gestational age at ANV1 (weeks) mean (SD) & $24.2(2.8)$ & $20.6(3.1)$ & $22.1(4.1)$ & 0.0001 \\
\hline Gestational age at delivery (weeks) mean (SD) & $39.6(1.9)$ & $39.4(2.7)$ & $39.3(3.2)$ & 0.15 \\
\hline \multicolumn{5}{|l|}{ Placental malaria (\%) } \\
\hline Positive & 3.0 & 11.4 & 9.2 & \multirow[t]{2}{*}{$<0.001$} \\
\hline Negative & 97.0 & 88.6 & 90.8 & \\
\hline \multicolumn{5}{|l|}{ Severe anaemia at ANV1 (\%) } \\
\hline Yes & 1.4 & 0.5 & 0.7 & \multirow[t]{2}{*}{0.05} \\
\hline No & 98.6 & 99.5 & 99.3 & \\
\hline \multicolumn{5}{|l|}{ Severe anaemia at delivery (\%) } \\
\hline Yes & 1.0 & 1.4 & 0.7 & \multirow[t]{2}{*}{0.4} \\
\hline No & 99.0 & 98.6 & 99.3 & \\
\hline Haemoglobin at ANV1 ( $\mathrm{g} / \mathrm{L}$ ) mean (SD) & $104.1(14.0)$ & $102.0(11.5)$ & $103.2(12.37)$ & 0.0004 \\
\hline Haemoglobin at ANV2 (g / L) mean (SD) & $105.2(13.4)$ & $102.1(10.5)$ & 105.1(10.8) & 0.0001 \\
\hline Haemoglobin at delivery $(\mathrm{g} / \mathrm{L}$ ) mean $(\mathrm{SD})$ & $113.7(16.3)$ & $110.0(14.0)$ & $111.5(14.4)$ & 0.0001 \\
\hline Number of antenatal visits mean (SD) & $3.1(0.7)$ & $3.7(1.3)$ & $3.5(1.0)$ & 0.0001 \\
\hline
\end{tabular}

SD: Standard deviation; BMI: Body Mass Index; ANV: Antenatal visit. 
multigravidae at each visit that did not reach significance. After pooling data from the three studies, both univariate and multivariate analyses showed a constant association between $\mathrm{Hb}$ concentrations and gravidity in the three periods of $\mathrm{Hb}$ assessment (Table 2). Mean $\mathrm{Hb}$ concentration was significantly lower in primigravidae than in multigravidae at ANV1 ( $<<0.001)$. Afterwards, it increased significantly in primigravidae only, with a tendency to reversal of the difference between primigravidae and multigravidae, which was confirmed at delivery $(\mathrm{p}<0.001)$, and an overall increase in all women.

The following covariates were associated to lower $\mathrm{Hb}$ levels at ANV1: gestational age more than or equal to 16 weeks $(\mathrm{P}<0.001)$, malaria $(\mathrm{P}<0.001)$, low $\mathrm{BMI}$ $(\mathrm{P}=0.001)$, study (STOPPAM and APEC studies $v s$ Ouidah study, $\mathrm{P}<0.001)$. At ANV2, malaria $(\mathrm{P}<0.001)$, low BMI (P < 0.001), study (STOPPAM and APEC studies vs Ouidah study, $\mathrm{P}<0.001$ ) were associated to lower $\mathrm{Hb}$ levels. At delivery, gestational age higher than or equal to 37 weeks $(P=0.005)$ and more than four ANVs during the follow-up $(\mathrm{P}=0.003)$ were associated with a higher $\mathrm{Hb}$ concentration, whereas malaria $(\mathrm{P}<0.001)$ and low BMI $(P=0.012)$ were related to lower Hb levels.

When maternal $\mathrm{Hb}$ was considered as a categorical variable (anaemia or no anaemia), primigravidity remained associated with a better $\mathrm{Hb}$ status at delivery $(\mathrm{aOR}=0.7, P=0.003)$ (Table 3$)$. The following covariates were related to an increased risk of maternal anaemia on ANV1: gestational age more than 16 weeks $(P=0.004)$, age below 21 years $(P=0.002)$, study (STOPPAM and APEC vs Ouidah study, $\mathrm{P}<0.001$ ), malaria $(\mathrm{P}<0.001)$ and low BMI $(\mathrm{P}<0.001)$. On ANV2, only STOPPAM study $(\mathrm{P}<0.001)$, malaria $(\mathrm{P}=0.001)$ and low BMI $(\mathrm{P}=0.01)$ were still associated with a higher risk for maternal anaemia. At delivery, a high number of ANVs (more than four) ( $\mathrm{P}=0.001$ ) was associated with a decreased risk for maternal anaemia, whereas malaria $(\mathrm{P}<0.001)$ was related to a higher risk of maternal anaemia.

Relationship between gravidity, malaria infection and iron deficiency throughout pregnancy

The relationships between the two main aetiologies of anaemia, malaria and iron deficiency, and gravidity are presented in Table 4. On ANV1 and delivery, malaria was more frequent in primigravidae, but the overall decrease of malaria between ANV1 and delivery was more important in primigravidae compared with multigravidae (more than $50 \%$ of reduction in primigravidae $v s$ less than $38 \%$ in multigravidae). Iron deficiency was assessed only in the APEC study. At ANV1, ANV2 and delivery, iron deficiency was more common in multigravidae, but the difference was only significant at ANV1 and ANV2.

\section{Discussion}

This study showed that if primigravidae were the most vulnerable group to anaemia at the beginning of pregnancy, they rapidly increased their $\mathrm{Hb}$ to reach higher levels than multigravidae at delivery. Protective interventions against malaria (i e, IPTp) appeared to play a major role in this process, which was demonstrated in three studies conducted in different areas at different times in southern Benin.

Although the women come from three different studies, they nevertheless share common factors. They all originated from the same region of Benin, with the same climatic pattern, and potentially similar malaria transmission in all sites [13]. They were all included before 28 weeks of gestation and they had the same average gestational age at delivery. Moreover, the women did not differ in the distribution of baseline characteristics such as age, body mass index at inclusion, which are prone to influence $\mathrm{Hb}$ concentrations [8]. However, although

Table 2 Relationship between mean haemoglobin $(\mathrm{Hb})$ and gravidity throughout pregnancy in south Benin

\begin{tabular}{|c|c|c|c|c|c|c|c|}
\hline \multirow[t]{2}{*}{ Gravidity } & \multirow[t]{2}{*}{$\mathrm{Hb}(\mathrm{g} / \mathrm{L})$} & \multicolumn{3}{|c|}{ Crude difference $^{*}(\mathrm{~g} / \mathrm{L})$} & \multicolumn{3}{|c|}{ Adjusted difference $^{* \delta}(\mathrm{g} / \mathrm{L})$} \\
\hline & & Mean & $\mathrm{Cl} 95 \%$ & $P$ value & Mean & $\mathrm{Cl} 95 \%$ & $P$ value \\
\hline \multicolumn{8}{|l|}{ ANV1 } \\
\hline Primigravidae $(n=786)$ & 100.9 & -3.1 & {$[-4.1,-2.0]$} & $<0.0001$ & -2.4 & {$[-3.4,-1.4]$} & $<0.001$ \\
\hline Multigravidae $(n=2733)$ & 104.0 & & & & & & \\
\hline \multicolumn{8}{|l|}{ ANV2 } \\
\hline Primigravidae $(n=721)$ & 105.0 & 0.8 & {$[-0.1,1.9]$} & 0.09 & 1.0 & {$[0.004,2.0]$} & 0.049 \\
\hline Multigravidae $(n=2520)$ & 104.2 & & & & & & \\
\hline \multicolumn{8}{|l|}{ Delivery } \\
\hline Primigravidae $(n=588)$ & 113.8 & 2.1 & {$[0.7,3.4]$} & 0.004 & 2.8 & {$[1.3,4.2]$} & $<0.001$ \\
\hline Multigravidae $(n=2241)$ & 111.7 & & & & & & \\
\hline
\end{tabular}

* Reference class is multigravidae.

${ }^{\S}$ Adjusted for malaria on the visit, BMI, number of ANVs, intervals between ANV1 and ANV2 and between ANV2 and delivery, schooling, study and gestational age. 
Table 3 Relationship between risk for maternal anaemia $(\mathrm{Hb}<110 \mathrm{~g} / \mathrm{L})$ and gravidity throughout pregnancy in south Benin

\begin{tabular}{|c|c|c|c|c|c|c|c|}
\hline \multirow[t]{2}{*}{ Gravidity } & \multirow[t]{2}{*}{$\%$ of anaemia } & \multicolumn{3}{|c|}{ Crude Odds ratio* } & \multicolumn{3}{|c|}{ Adjusted Odds ratio*\$ } \\
\hline & & OR & $\mathrm{Cl} 95 \%$ & $P$ value & OR & $\mathrm{Cl} 95 \%$ & $P$ value \\
\hline \multicolumn{8}{|l|}{ ANV1 } \\
\hline Primigravidae $(n=786)$ & 73.9 & 1.4 & {$[1.1,1.6]$} & 0.001 & 1.1 & {$[0.9,1.4]$} & 0.28 \\
\hline Multigravidae $(n=2733)$ & 68.0 & & & & & & \\
\hline \multicolumn{8}{|l|}{ ANV2 } \\
\hline Primigravidae $(n=721)$ & 66.0 & 0.9 & {$[0.8,1.1]$} & 0.44 & & & \\
\hline Multigravidae $(n=2520)$ & 67.5 & & & & & & \\
\hline \multicolumn{8}{|l|}{ Delivery } \\
\hline Primigravidae $(n=588)$ & 36.9 & 0.8 & {$[0.7,0.9]$} & 0.02 & 0.7 & {$[0.6,0.9]$} & 0.003 \\
\hline Multigravidae $(n=2241)$ & 42.4 & & & & & & \\
\hline
\end{tabular}

*Reference class is multigravidae.

${ }^{\S}$ Adjusted for malaria on the visit, BMI, number of ANVs, intervals between ANV1 and ANV2 and between ANV2 and delivery, schooling, study and gestational age.

all women received two doses of IPTp with a minimal one-month interval between intakes, the study designs were different: STOPPAM being an observational study and Ouidah and APEC controlled randomized trials. The timing of IPTp also differed between the three studies, the last intake being on average one month closer to delivery in Ouidah compared with the two other studies and probably related to a lower prevalence of placental infection, as stressed by Huynh et al [14]. STOPPAM study and APEC trial were located in more rural settings than Ouidah. Finally, investigations were held at different times and one can assume that, in particular, resistance of malaria parasites to SP may have progressed from 2005 until now.

The analyses of data from each of the three studies separately showed that primigravidae who were initially at higher risk of anaemia at inclusion (ANV1), increased progressively $\mathrm{Hb}$ concentration and became, although not significantly, at lower risk of anaemia at delivery

Table 4 Relationship between gravidity, malaria infections and iron deficiency throughout pregnancy in south Benin, univariate analyses

\begin{tabular}{llllllll}
\hline Gravidity & \multicolumn{3}{c}{ Malaria infections* } & & \multicolumn{3}{c}{ Iron deficiency $^{\S}$} \\
\cline { 2 - 3 } \cline { 5 - 7 } & $\mathrm{n}$ & $\%$ & $P$ value & & $\mathrm{n}$ & $\%$ & $P$ value \\
\hline
\end{tabular}

ANV1

\begin{tabular}{|c|c|c|c|c|c|c|}
\hline Primigravidae & 791 & 20.2 & $<0.001$ & 190 & 23.7 & 0.002 \\
\hline Multigravidae & 2751 & 9.6 & & 815 & 35.6 & \\
\hline \multicolumn{7}{|l|}{ NV2 } \\
\hline Primigravidae & 727 & 3.8 & 0.44 & 180 & 26.7 & 0.003 \\
\hline Multigravidae & 2547 & 3.3 & & 765 & 38.4 & \\
\hline \multicolumn{7}{|l|}{ elivery } \\
\hline Primigravidae & 617 & 9.1 & 0.005 & 155 & 27.7 & 0.4 \\
\hline Multigravidae & 2328 & 6.0 & & 701 & 31.2 & \\
\hline
\end{tabular}

*Data from the three studies.

${ }^{5}$ Data from APEC only (Iron status was only assessed in APEC study). compared with multigravidae. Pooling all data from the three studies increased the sample size and thus the power of the analysis, demonstrating the importance of gravidity as a determinant of maternal anaemia, even after adjusting on the characteristics of each population. In addition, taking into account gestational age in the multivariate analyses minimized the effect of potential confounders such as the gestational plasma volume changes that might as well have played a role in the parity-related difference.

Primigravidae had a lower mean $\mathrm{Hb}$ and an increased risk for anaemia in early pregnancy compared to multigravidae, prior to the administration of IPTp. Furthermore, an overall decrease in the proportion of malaria infections after women were given IPTp has been shown [1,15-17]. In the study, more than $20 \%$ of primigravidae were infected by malarial parasites at inclusion, whereas only less than $10 \%$ of them were malaria positive at delivery. At the same time, the proportion of malariainfected multigravidae decreased from $10 \%$ to $6 \%$. The decline in the proportion of malaria infections in primigravidae also coincides with the increase of $\mathrm{Hb}$ concentrations on ANV2 and delivery. Such an increase of $\mathrm{Hb}$ concentrations in primigravidae may then be explained by the reduction of the proportion of malaria-related anaemia by IPTp in this group. These results are in agreement with a large meta-analysis of $\mathrm{Hb}$ parity differences comparing malarious and non malarious areas [18]. In addition, the demonstration of a better efficacy of anti-malarial drugs in primigravidae for the prevention of anaemia had been made in the 1990s, when chloroquine chemoprophylaxis was still used [19]. Since the implementation of IPTp with SP, two observational studies led to conflicting results [20,21]. Rogerson et al showed in Malawian women a decrease of maternal anaemia only in primigravidae [20], whereas Hommerich et al showed a decrease of anaemia in only 
Ghanaian multigravidae [21]. Finally, a Cochrane review of the effects of drugs to prevent malaria-related illnesses in pregnant women concluded that IPTp was effective to reduce the risk for peripheral and placental malaria and maternal anaemia, especially in primi and secundigravidae [22].

In spite of IPTp and iron supplementation, multigravidae increased $\mathrm{Hb}$ levels, but not in the same proportion as primigravidae, and consequently were at higher risk of anaemia at delivery. Indeed, in the APEC study, the proportion of women presenting with iron deficiency was higher in multigravidae than in primigravidae at each blood assessment. As previously described [1], there is a great demand for iron during pregnancy, as shown by the drop in $\mathrm{Hb}$ levels in the second trimester of gestation. Consequently, the effect of iron supplementation may be masked and even be insufficient to cover the needs of the mother and the foetus. In agreement with this hypothesis, Hernandez-Martinez et al showed in Spanish, well-nourished, pregnant women (including primi and multigravidae) who received an iron supplementation (40-60 mg/day) that iron deficiency increased importantly as pregnancy progressed (from $8 \%$ at inclusion to $68 \%$ at delivery) [23]. A similar trend was not observed in APEC. On the contrary, iron deficiency seemed to decrease in multigravidae (from 35\% at ANV1 to $31 \%$ at delivery), suggesting that the effect of iron supplementation might be better than expected. In this study, the difference between primigravidae and multigravidae may be a consequence of an increase in the prevalence of iron deficiency, due to cumulative iron requirements of successive pregnancies. Additionally, closely spaced pregnancies that are frequently observed in sub-Saharan Africa may exacerbate this phenomenon, as birth intervals have been found to affect women's $\mathrm{Hb}$ concentrations, with short intervals being a risk factor for anaemia [24].

The lack of longitudinal data for pregnant women with the same definition of iron deficiency makes comparisons of prevalence of iron deficiency difficult among studies from sub-Saharan Africa. Nevertheless, in Kenyan pregnant women of less than 24 weeks' gestational age, results close to the findings of this study were found by Alusala et al [25]. The authors showed that more than $40 \%$ of multigravidae were iron deficient, defined as serum ferritin concentrations below $12 \mu \mathrm{g} / \mathrm{L}$ vs less than $19 \%$ of primigravidae. This is in agreement with the results of APEC (36\% and $24 \%$ in the two groups).

\section{Conclusion}

In the context of IPTp, primigravidae were shown to have a progressive increase in $\mathrm{Hb}$ concentration throughout pregnancy. The effect of IPTp on anaemia in multigravidae women was less marked, as they are less susceptible to malaria and nutritional deficiencies seem to be the main causes of anaemia in this group. There is a need to reinforce malaria prevention strategies in both groups, and to undertake additional measures focusing on the reduction of micronutrient deficiencies in multigravidae. For instance, women should be encouraged to take iron supplements from the first pregnancy until menopause, even at lower doses during the interval between pregnancies, to reduce iron side effects and increase compliance. This strategy is relatively easy to implement as the target population may be identified at the first pregnancy.

\section{Competing interests}

The authors declare that they have no competing interests.

\section{Authors' contributions}

SO participated to conceive APEC study, participated in its design and coordination, performed statistical analyses and drafted and finalized the manuscript. FBL participated in statistical analyses and the finalization of the manuscript. VB participated to conceive Ouidah study, participated in its design and coordination, participated to the statistical analyses of the manuscript. BTH participated to implement and coordinate STOPPAM study and the finalization of the manuscript. GKK participated to implement APEC study, in the statistical analyses and the finalization of the manuscript. MMKA participated to the implementation and coordination of APEC study. NF participated to conceive, implement and coordinate STOPPAM study and the finalization of the manuscript. AM participated to conceive, implement and coordinate STOPPAM study and the finalization of the manuscript. PD participated to conceive and coordinate STOPPAM study and the finalization of the manuscript. MC participated to conceive and coordinate the three studies (Ouidah, STOPPAM, APEC), in the statistical analyses and the finalization of the manuscript. All authors read and approved the final manuscript.

\section{Acknowledgements}

We thank the women who participated in the three studies. We also thank the midwives of the district of Ouidah, Comé and Allada and their assistants for their help in conducting these studies. The Ouidah clinical trial was founded by French Institute of Research for Development (IRD). STOPPAM is a small and medium-scale collaborative project supported by the European Seventh Framework Programme, Contract Number: 200889. APEC study was supported by the Malaria in Pregnancy (MiP) Consortium, which is funded through a grant from the Bill and Melinda Gates Foundation to the Liverpool School of Tropical Medicine. MiPPAD trial is co-funded by the EDCTP (EDCTP- IP.07.31080.002). We also thank MiPPAD Executive Committee and $\mathrm{MiPc}$ reviewers for valuable inputs in this work. Smaïla Ouédraogo was supported by an IRD grant while writing this paper.

\section{Author details}

'Mère et enfant face aux infections tropicales, IRD Unité mixte de recherche 216, Paris, France. 'Ecole des Hautes Etudes en Santé Publique, Rennes, France. ${ }^{3}$ Laboratoire de Parasitologie, Faculté des Sciences de la Santé, Cotonou, Benin. ${ }^{4}$ PRES Sorbonne Paris Cité, Faculté de pharmacie, Paris, France.

Received: 26 June 2012 Accepted: 18 October 2012 Published: 22 October 2012

\section{References}

1. Ouédraogo S, Koura GK, Bodeau-Livinec F, Accrombessi MMK, Massougbodji A, Cot M: Maternal anaemia in pregnancy: assessing the effect of preventive measures in a malaria endemic area. Am J Trop Med Hyg, in press.

2. Brabin BJ: An analysis of malaria in pregnancy in Africa. Bull World Health Organ 1983, 61:1005-1016. 
3. Shulman CE, Graham WJ, Jilo H, Lowe BS, New L, Obiera J, Snow RW, Marsh K: Malaria is an important cause of anaemia in primigravidae: evidence from a district hospital in coastal Kenya. Trans $R$ Soc Trop Med Hyg 1996, 90:535-539.

4. Ndyomugyenyi R, Magnussen P: Anaemia in pregnancy: Plasmodium falciparum infection is an important cause in primigravidae in Hoima district, western Uganda. Ann Trop Med Parasitol 1999, 93:457-465.

5. Brabin BJ, Ginny M, Sapau J, Galme K, Paino J: Consequences of maternal anaemia on outcome of pregnancy in a malaria endemic area in Papua New Guinea. Ann Trop Med Parasitol 1990, 84:11-24.

6. Briand V, Bottero J, Noel H, Masse V, Cordel H, Guerra J, Kossou H, Fayomi B, Ayemonna P, Fievet N, Massougbodji A, Cot M: Intermittent treatment for the prevention of malaria during pregnancy in Benin: a randomized, open-label equivalence trial comparing sulfadoxine-pyrimethamine with mefloquine. J Infect Dis 2009, 200:991-1001.

7. Huynh BT, Fievet N, Gbaguidi G, Dechavanne S, Borgella S, Guezo-Mevo B, Massougbodji A, Ndam NT, Deloron P, Cot M: Influence of the timing of malaria infection during pregnancy on birth weight and on maternal anemia in Benin. Am J Trop Med Hyg 2011, 85:214-220.

8. Ouédraogo S, Koura GK, Accrombessi MMK, Bodeau-Livinec F, Massougbodji A, Cot M: Maternal anemia at first antenatal visit: prevalence and risk factors in a malaria-endemic area in Benin. Am J Trop Med Hyg 2012, 87:418-24.

9. Greenwood BM, Armstrong JR: Comparison of two simple methods for determining malaria parasite density. Trans R Soc Trop Med Hyg 1991, 85:186-188.

10. Planche T, Krishna S, Kombila M, Engel K, Faucher JF, Ngou-Milama E, Kremsner PG: Comparison of methods for the rapid laboratory assessment of children with malaria. Am J Trop Med Hyg 2001, 65:599-602

11. WHO: The prevalence of anaemia in women. A tabulation of available information. 2nd edition. Geneva: WHO; 1992.

12. Bodeau-Livinec F, Briand V, Berger J, Xiong X, Massougbodji A, Day KP, Cot M: Maternal anemia in benin: prevalence, risk factors, and association with low birth weight. Am J Trop Med Hyg 2011, 85:414-420.

13. Moiroux N, Boussari O, Djenontin A, Damien G, Cottrell G, Henry MC, Guis H, Corbel V: Dry season determinants of malaria disease and net use in Benin. West Africa PLoS One 2012, 7:e30558.

14. Huynh BT, Fievet N, Briand B, Borgella S, Massougbodji A, Deloron P, Cot M: Consequences of gestational malaria on birth weight: finding the best timeframe for intermittent preventive treatment administration. PLOS One 2012, 7:e35342.

15. Kayentao K, Kodio M, Newman RD, Maiga H, Doumtabe D, Ongoiba A Coulibaly D, Keita AS, Maiga B, Mungai M, Parise ME, Doumbo O: Comparison of intermittent preventive treatment with chemoprophylaxis for the prevention of malaria during pregnancy in Mali. J Infect Dis 2005, 191:109-116.

16. Schultz LJ, Steketee RW, Macheso A, Kazembe P, Chitsulo L, Wirima JJ: The efficacy of antimalarial regimens containing sulfadoxine-pyrimethamine and/or chloroquine in preventing peripheral and placental Plasmodium falciparum infection among pregnant women in Malawi. Am J Trop Med Hyg 1994, 51:515-522.

17. Parise ME, Ayisi JG, Nahlen BL, Schultz LJ, Roberts JM, Misore A, Muga R, Oloo AJ, Steketee RW: Efficacy of sulfadoxine-pyrimethamine for prevention of placental malaria in an area of Kenya with a high prevalence of malaria and human immunodeficiency virus infection. Am J Trop Med Hyg 1998, 59:813-822.

18. Savage EJ, Msyamboza K, Gies S, D'Alessandro U, Brabin BJ: Maternal anaemia as an indicator for monitoring malaria control in pregnancy in sub-Saharan Africa. BJOG 2007, 114:1222-1231.

19. Cot M, le Hesran JY, Miailhes P, Roisin A, Fievet N, Barro D, Etya'Ale D, Deloron P, Carnevale P, Breart G: Effect of chloroquine prophylaxis during pregnancy on maternal haematocrit. Ann Trop Med Parasitol 1998, 92:37-43.

20. Rogerson SJ, Chaluluka E, Kanjala M, Mkundika P, Mhango C, Molyneux ME: Intermittent sulfadoxine-pyrimethamine in pregnancy: effectiveness against malaria morbidity in Blantyre, Malawi, in 1997-99. Trans $R$ Soc Trop Med Hyg 2000, 94:549-553.

21. Hommerich L, von Oertzen C, Bedu-Addo G, Holmberg V, Acquah PA, Eggelte TA, Bienzle U, Mockenhaupt FP: Decline of placental malaria in southern Ghana after the implementation of intermittent preventive treatment in pregnancy. Malar J 2007, 6:144.

22. Garner P, Gulmezoglu AM: Drugs for preventing malaria-related illness in pregnant women and death in the newborn. Cochrane Database Syst Rev 2003, 4:CD000169.

23. Hernandez-Martinez C, Canals J, Aranda N, Ribot B, Escribano J, Arija V: Effects of iron deficiency on neonatal behavior at different stages of pregnancy. Early Hum Dev 2011, 87:165-169.

24. Conde-Agudelo A, Belizán JM: Maternal morbidity and mortality associated with interpregnancy interval: cross sectional study. BMJ 2000, 321:1255-1259.

25. Alusala DN, Estambale BB, Magnussen P, Friis H, Luoba Al, Mwaniki D: Predictors of serum ferritin and haemoglobin during pregnancy, in a malaria-endemic area of western Kenya. Ann Trop Med Parasitol 2008, 102:297-308.

doi:10.1186/1475-2875-11-348

Cite this article as: Ouédraogo et al:: Malaria and gravidity interact to modify maternal haemoglobin concentrations during pregnancy.

Malaria Journal 2012 11:348.

\section{Submit your next manuscript to BioMed Central and take full advantage of:}

- Convenient online submission

- Thorough peer review

- No space constraints or color figure charges

- Immediate publication on acceptance

- Inclusion in PubMed, CAS, Scopus and Google Scholar

- Research which is freely available for redistribution 